\title{
Cyclic C-Amino Phosphorus Ylides as a Source of Bidentate Heteroditopic Ligands (Phosphine I Aminocarbene) for Transition Metals
}

Joan Vignolle, Bruno Donnadieu, Didier Bourissou, Michèle Soleilhavoup, and Guy Bertrand*

UCR-CNRS Joint Research Chemistry Laboratory (UMI 2957), Department of Chemistry, University of California Riverside, Riverside CA 92521-0403, and Laboratoire Hétérochimie Fondamentale et Appliquée du CNRS (UMR 5069) Université Paul Sabatier, 118, route de Narbonne, F-31062 Toulouse Cedex 09 (France)

\section{Supporting Information}




\section{Synthesis and Spectroscopic Data}

All manipulations were performed under an inert atmosphere of argon using standard Schlenk techniques. Dry, oxygen-free solvents were employed. ${ }^{1} \mathrm{H},{ }^{13} \mathrm{C}$ and ${ }^{31} \mathrm{P}$ NMR spectra were recorded on Varian Inova 300, 500 and Bruker Avance 300, 400 and 500 spectrometers.

Synthesis of Pd(II) complex 2. A THF solution (5 mL) of ylide $\mathbf{1}^{1}(0.16 \mathrm{~g}, 0.59$ mmol) was added at $-78{ }^{\circ} \mathrm{C}$ to a THF solution $(8 \mathrm{~mL})$ of dichloro(1,5cyclooctadiene)palladium $(0.17 \mathrm{~g}, 0.59 \mathrm{mmol})$. The suspension was stirred at room temperature for $12 \mathrm{~h}$. After evaporation of the solvent under vacuum, the residue was washed with $\mathrm{Et}_{2} \mathrm{O}(2 \times 10 \mathrm{~mL})$, and was then dissolved in boiling THF. Complex 2 crystallized at room temperature from the THF solution, and was isolated as pale yellow crystals (0.19 g, 81\%): mp 143-144 ${ }^{\circ} \mathrm{C}(\mathrm{dec}) ;{ }^{31} \mathrm{P}$ NMR $\left(\mathrm{CD}_{3} \mathrm{CN}, 25{ }^{\circ} \mathrm{C}\right) \delta+$ 98.1; ${ }^{1} \mathrm{H}$ NMR $\left(\mathrm{CD}_{3} \mathrm{CN}, 25{ }^{\circ} \mathrm{C}\right) \delta 1.24\left(\mathrm{~d}, 6 \mathrm{H}, J_{\mathrm{HH}}=6.7 \mathrm{~Hz}, \mathrm{CHCH}_{3}\right), 1.45(\mathrm{~d}, 6 \mathrm{H}$, $\left.J_{\mathrm{HH}}=6.8 \mathrm{~Hz}, \mathrm{CHCH}_{3}\right), 2.67\left(\mathrm{~d}, 18 \mathrm{H}, J_{\mathrm{PH}}=10.3 \mathrm{~Hz}, \mathrm{NCH}_{3}\right), 4.05$ (sept, $1 \mathrm{H}, J_{\mathrm{HH}}=6.7$ $\mathrm{Hz}, \mathrm{CHCH}_{3}$ ), 5.35 (sept, $\left.1 \mathrm{H}, J_{\mathrm{HH}}=6.8 \mathrm{~Hz}, \mathrm{CHCH}_{3}\right), 10.03\left(\mathrm{~d}, 1 \mathrm{H}, J_{\mathrm{PH}}=7.8 \mathrm{~Hz}\right.$, $\mathrm{NCH}) ;{ }^{13} \mathrm{C}$ NMR $\left(\mathrm{CD}_{3} \mathrm{CN}, 25{ }^{\circ} \mathrm{C}\right) \delta 20.0\left(\mathrm{~s}, \mathrm{CHCH}_{3}\right), 24.5\left(\mathrm{~s}, \mathrm{CHCH}_{3}\right), 39.2\left(\mathrm{~d}, \mathrm{~J}_{\mathrm{PC}}=\right.$ $6.3 \mathrm{~Hz}, \mathrm{NCH}_{3}$ ), 54.2 (s, $\left.\mathrm{CHCH}_{3}\right), 68.7\left(\mathrm{~s}, \mathrm{CHCH}_{3}\right), 213.9$ (d, $J_{\mathrm{PC}}=14.3 \mathrm{~Hz}, \mathrm{NCH}$ ); MS (FAB+) $419\left[\mathrm{M}^{+}-\mathrm{Cl}\right]$.

Synthesis of Pd(II) complex 4. A THF solution (10 mL) of the phosphonium salt $\mathbf{3}^{1}$ $(0.40 \mathrm{~g}, 0.70 \mathrm{mmol})$ was cooled at $-78{ }^{\circ} \mathrm{C}$ and 1 eq of LiHMDS $(0.12 \mathrm{~g}, 0.70 \mathrm{mmol})$ in $5 \mathrm{~mL}$ THF was added. The solution was warmed to room temperature and stirred for 1 h. This reaction mixture was added to a THF solution $(10 \mathrm{~mL})$ of dichloro(1,5cyclooctadiene)palladium $(0.20 \mathrm{~g}, 0.70 \mathrm{mmol})$ at $-78{ }^{\circ} \mathrm{C}$. The solution was stirred at room temperature for $12 \mathrm{~h}$. After filtration, and recrystallization in $\mathrm{CH}_{2} \mathrm{Cl}_{2}$ /hexane at room temperature, the carbene complex 4 was isolated as yellow crystals $(0.25 \mathrm{~g}$, 60\%): mp 132-133 ${ }^{\circ} \mathrm{C}$ (dec); ${ }^{31} \mathrm{P} \mathrm{NMR}\left(\mathrm{CDCl}_{3}, 25{ }^{\circ} \mathrm{C}\right) \delta+4.6 ;{ }^{1} \mathrm{H} \mathrm{NMR}\left(\mathrm{CDCl}_{3}, 25\right.$ $\left.{ }^{\circ} \mathrm{C}\right) \delta 0.60\left(\mathrm{~d}, 3 \mathrm{H}, J_{\mathrm{HH}}=6.8 \mathrm{~Hz}, \mathrm{CHCH}_{3}\right), 0.88\left(\mathrm{~d}, 3 \mathrm{H}, J_{\mathrm{HH}}=7.5 \mathrm{~Hz}, \mathrm{CHCH}_{3}\right), 1.40$ $\left(\mathrm{d}, 3 \mathrm{H}, J_{\mathrm{HH}}=7.0 \mathrm{~Hz}, \mathrm{CHCH}_{3}\right), 1.65\left(\mathrm{~d}, 3 \mathrm{H}, J_{\mathrm{HH}}=7.0 \mathrm{~Hz}, \mathrm{CHCH}_{3}\right), 1.86\left(\mathrm{~s}, 3 \mathrm{H}, \mathrm{CH}_{3}\right)$, 3.02 (s, 3H, CH $\mathrm{CH}_{3}, 4.59$ (sept, $1 \mathrm{H}, J_{\mathrm{HH}}=7.0 \mathrm{~Hz}, \mathrm{CHCH}_{3}$ ), 6.01 (br, $1 \mathrm{H}, \mathrm{CHCH}_{3}$ ), 7.32-7.41 (m, 4H, $\left.\mathrm{CH}_{\mathrm{ar}}\right)$, 7.45-7.62 (m, 8H, $\left.\mathrm{CH}_{\mathrm{ar}}\right), 8.01-8.08\left(\mathrm{~m}, 2 \mathrm{H}, \mathrm{CH}_{\mathrm{ar}}\right) ;{ }^{13} \mathrm{C} \mathrm{NMR}$ $\left(\mathrm{CDCl}_{3}, 25{ }^{\circ} \mathrm{C}\right) \delta 21.8\left(\mathrm{~s}, \mathrm{CHCH}_{3}\right), 21.9\left(\mathrm{~s}, \mathrm{CHCH}_{3}\right), 23.9\left(\mathrm{~s}, \mathrm{CHCH}_{3}\right), 25.3$ $\left.\left(\mathrm{s}, \mathrm{CHCH}_{3}\right)\right), 44.3\left(\mathrm{~s}, \mathrm{C}\left(\mathrm{CH}_{3}\right)_{2}\right), 51.0\left(\mathrm{~d}, J_{\mathrm{CP}}=10.5 \mathrm{~Hz}, \mathrm{C}\left(\mathrm{CH}_{3}\right)_{2}\right), 59.5\left(\mathrm{~s}, \mathrm{CHCH}_{3}\right)$, $124.0\left(\mathrm{~d}, J_{\mathrm{CP}}=50.4 \mathrm{~Hz}, C_{\mathrm{ar}}\right), 127.7\left(\mathrm{~s}, C_{\mathrm{ar}}\right), 128.5$ (d, $\left.J_{\mathrm{CP}}=99.2 \mathrm{~Hz}, C_{\mathrm{ar}}\right), 128.6$ (d, $\left.J_{\mathrm{CP}}=12.2 \mathrm{~Hz}, C \mathrm{H}_{\mathrm{ar}}\right), 129.4$ (d, $\left.J_{\mathrm{CP}}=10.5 \mathrm{~Hz}, C \mathrm{H}_{\mathrm{ar}}\right), 131.2\left(\mathrm{~s}, C \mathrm{H}_{\mathrm{ar}}\right), 132.0\left(\mathrm{~s}, C \mathrm{H}_{\mathrm{ar}}\right)$, $132.1\left(\mathrm{~d}, J_{\mathrm{CP}}=56.5 \mathrm{~Hz}, C_{\mathrm{ar}}\right), 133.3\left(\mathrm{~s}, C \mathrm{H}_{\mathrm{ar}}\right), 133.7$ (d, $\left.J_{\mathrm{CP}}=10.7 \mathrm{~Hz}, C \mathrm{H}_{\mathrm{ar}}\right), 134.7$ (d, $\left.J_{\mathrm{CP}}=10.7 \mathrm{~Hz}, C_{\mathrm{ar}}\right), 138.5\left(\mathrm{~s}, C_{\mathrm{ar}}\right), 150.9\left(\mathrm{~d}, J_{\mathrm{CP}}=12.2 \mathrm{~Hz}, C_{\mathrm{ar}}\right), 247.3$ (s, $C_{\text {carbene }}$ ); MS (FAB+) $558\left[\mathrm{M}^{+}-\mathrm{Cl}\right]$.

Synthesis of 2-formyl-2'-diphenylphosphinobiphenyl: A Schlenk tube was charged with 2-bromo-2'-formylbiphenyl ${ }^{2}$ (11.0 g, 42.1 mmol), $\mathrm{Pd}\left(\mathrm{PPh}_{3}\right)_{4}(0.36 \mathrm{~g}, 0.31 \mathrm{mmol})$ and $60 \mathrm{~mL}$ of toluene. Then, diphenylphosphine $(8.8 \mathrm{~mL}, 50.5 \mathrm{mmol})$ and triethylamine $(5.9 \mathrm{~mL}, 42.1 \mathrm{mmol})$ were added successively at room temperature and the mixture was refluxed for 3 days. Ammonium salts were filtered off and the red 
solution was washed with $3 \times 30 \mathrm{~mL}$ of a degassed aqueous $\mathrm{NH}_{4} \mathrm{Cl}$ solution and with $50 \mathrm{~mL}$ of a degassed aqueous $\mathrm{NaCl}$ solution. The solvent was evaporated under vacuum affording a red oily residue. $20 \mathrm{~mL}$ of $\mathrm{Et}_{2} \mathrm{O}$ was added and the resulting heterogeneous mixture was filtered. The solid was washed with $5 \mathrm{x} 2 \mathrm{~mL}$ of $\mathrm{Et}_{2} \mathrm{O}$. The product was isolated as a white powder (7.5 g, 48\%): $\mathrm{mp} 115-116{ }^{\circ} \mathrm{C} ;{ }^{31} \mathrm{P}$ NMR $\left(\mathrm{C}_{6} \mathrm{D}_{6}\right.$, $\left.25{ }^{\circ} \mathrm{C}\right) \delta-12,1 ;{ }^{1} \mathrm{H}$ NMR $\left(\mathrm{C}_{6} \mathrm{D}_{6}, 25{ }^{\circ} \mathrm{C}\right) \delta$ 7.04-7.35 (m, 17H, $\left.\mathrm{CH}_{\mathrm{ar}}\right), 8.13(\mathrm{~m}, 1 \mathrm{H}$, $\left.\mathrm{CH}_{\mathrm{ar}}\right), 10.08(\mathrm{~s}, \mathrm{CHO}) ;{ }^{13} \mathrm{C}\left(\mathrm{C}_{6} \mathrm{D}_{6}, 25{ }^{\circ} \mathrm{C}\right) \delta 127.5\left(\mathrm{~s}, \mathrm{CH}_{\mathrm{ar}}\right), 128.6\left(\mathrm{~s}, \mathrm{CH}_{\mathrm{ar}}\right), 128.8(\mathrm{~s}$, $C_{\mathrm{ar}}$ ), 129.0 (d, $J_{\mathrm{CP}}=6.8 \mathrm{~Hz}, C_{\mathrm{ar}}$ ), 129.1 (d, $\left.J_{\mathrm{CP}}=8.3 \mathrm{~Hz}, C \mathrm{H}_{\mathrm{ar}}\right), 129.2\left(\mathrm{~s}, C \mathrm{H}_{\mathrm{ar}}\right.$ ), 129.6 (s, $C \mathrm{H}_{\mathrm{ar}}$ ), 131.5 (d, $J_{\mathrm{CP}}=4.5 \mathrm{~Hz}, C_{\mathrm{ar}}$ ), 132.2 (d, $J_{\mathrm{CP}}=3.0 \mathrm{~Hz}, C_{\mathrm{ar}}$ ), 132.9 (s, $\mathrm{CH}_{\mathrm{ar}}$ ), 133.8 (s, $\mathrm{CH}_{\mathrm{ar}}$ ), 134.4 (s, $C_{\mathrm{ar}}$ ), 134.7 (d, $J_{\mathrm{CP}}=20.0 \mathrm{~Hz}, \mathrm{CH}_{\mathrm{ar}}$ ), 135.0 (d, $J_{\mathrm{CP}}=$ $\left.20.7 \mathrm{~Hz}, C \mathrm{H}_{\mathrm{ar}}\right), 136.6\left(\mathrm{~d}, J_{\mathrm{CP}}=11.9 \mathrm{~Hz}, C_{\mathrm{ar}}\right), 137.1\left(\mathrm{~d}, J_{\mathrm{CP}}=12.1 \mathrm{~Hz}, C_{\mathrm{ar}}\right), 138.6(\mathrm{~d}$, $\left.J_{\mathrm{CP}}=14.8 \mathrm{~Hz}, C_{\mathrm{ar}}\right), 144.1\left(\mathrm{~d}, J_{\mathrm{CP}}=28.1 \mathrm{~Hz}, C_{\mathrm{ar}}\right), 145.2\left(\mathrm{~d}, J_{\mathrm{CP}}=6.0 \mathrm{~Hz}, C_{\mathrm{ar}}\right), 190.7$ $\left(\mathrm{d}, J_{\mathrm{CP}}=2.4 \mathrm{~Hz}, \mathrm{CHO}\right) ; \mathrm{MS}\left(\mathrm{DCI} / \mathrm{NH}_{3}\right) 367[\mathrm{M}+\mathrm{H}]^{+}$

Synthesis of phosphonium salt 5: To a solution of 2-formyl-2'diphenylphosphinobiphenyl (3.93 g, $10.73 \mathrm{mmol}$ ) in $20 \mathrm{~mL}$ toluene, $\mathrm{N}$ trimethylsilylpiperidine $(3.0 \mathrm{~mL}, 16.1 \mathrm{mmol})$ was added at room temperature. The solution was heated at $100^{\circ} \mathrm{C}$ for two days and then cooled to room temperature. After adding $\mathrm{Me}_{3} \mathrm{SiOTf}(2.3 \mathrm{~mL}, 12.9 \mathrm{mmol})$ at $0^{\circ} \mathrm{C}$, the product precipitated immediately. This suspension was stirred for $30 \mathrm{~min}$ at $0^{\circ} \mathrm{C}$ and a further hour at room temperature. The solution was filtered off and the solid was washed with $2 \mathrm{x} 20 \mathrm{~mL}$ of $\mathrm{Et}_{2} \mathrm{O}, 2 \mathrm{x} 10$ $\mathrm{mL}$ of a mixture $\mathrm{Et}_{2} \mathrm{O} / \mathrm{THF}(1 / 1)$ and with $20 \mathrm{~mL}$ of THF. After evaporation of the residual solvent, the phosphonium salt 5 was obtained as a white powder (1.9 g, 30\%): mp 225-227 ${ }^{\circ} \mathrm{C} ;{ }^{31} \mathrm{P} \mathrm{NMR}\left(\mathrm{CDCl}_{3}, 25{ }^{\circ} \mathrm{C}\right) \delta+4.1 ;{ }^{1} \mathrm{H} \mathrm{NMR}\left(\mathrm{CDCl}_{3}, 25{ }^{\circ} \mathrm{C}\right) \delta$ $1.23\left(\mathrm{~m}, 2 \mathrm{H}, \mathrm{CH}_{2}\right.$ ), 1.29 (br, $4 \mathrm{H}, \mathrm{CH}_{2}$ ), 2.36 (br, 4H, $\mathrm{CH}_{2}$ ), 5.53 (d, $1 \mathrm{H}, J_{\mathrm{PH}}=7.3 \mathrm{~Hz}$, CHP), $7.31\left(\mathrm{t}_{\text {like, }}, 1 \mathrm{H}, J_{\mathrm{HH}}=7.4 \mathrm{~Hz}, \mathrm{CH}_{\mathrm{ar}}\right), 7.42$ (t like, $\left.1 \mathrm{H}, J_{\mathrm{HH}}=7.7 \mathrm{~Hz}, \mathrm{CH}_{\mathrm{ar}}\right), 7.45-$ $7.80\left(\mathrm{~m}, 14 \mathrm{H}, \mathrm{CH}_{\mathrm{ar}}\right), 7.98\left(\mathrm{t}_{\text {like }}, 1 \mathrm{H}, J_{\mathrm{HH}}=7.7 \mathrm{~Hz}, \mathrm{CH}_{\mathrm{ar}}\right), 8.09$ (dd, $1 \mathrm{H}, J_{\mathrm{HH}}=7.4 \mathrm{~Hz}$, $\left.J_{\mathrm{PH}}=7.4 \mathrm{~Hz}, \mathrm{CH}_{\mathrm{ar}}\right) ;{ }^{13} \mathrm{C} \mathrm{NMR}\left(\mathrm{CDCl}_{3}, 25{ }^{\circ} \mathrm{C}\right) \delta 22.0\left(\mathrm{~s}, \mathrm{CH}_{2}\right), 26.0\left(\mathrm{~s}, \mathrm{CH}_{2}\right), 53.3(\mathrm{~s}$, $\left.C_{2}\right), 61.8\left(\mathrm{~d}, J_{\mathrm{CP}}=69.6 \mathrm{~Hz}, C H P\right), 112.9\left(\mathrm{~d}, J_{\mathrm{CP}}=84.6 \mathrm{~Hz}, C_{\mathrm{ar}}\right), 115.3\left(\mathrm{~d}, J_{\mathrm{CP}}=\right.$ $\left.105.0 \mathrm{~Hz}, C_{\mathrm{ar}}\right), 116.1$ (d, $J_{\mathrm{CP}}=85.7 \mathrm{~Hz}, C_{\mathrm{ar}}$ ), 121.0 (q, $J_{\mathrm{CF}}=320.9 \mathrm{~Hz}, C_{3} \mathrm{SO}_{3}$ ), 125.5 (s, $\left.C_{\mathrm{ar}}\right), 127.3\left(\mathrm{~s}, C_{\mathrm{ar}}\right), 127.9$ (d, $\left.J_{\mathrm{CP}}=9.5 \mathrm{~Hz}, C_{\mathrm{ar}}\right), 129.6\left(\mathrm{~d}, J_{\mathrm{CP}}=11.5 \mathrm{~Hz}, C_{\mathrm{ar}}\right), 130.0$ (br, $\left.C_{\mathrm{ar}}\right), 130.3\left(\mathrm{~s}, C_{\mathrm{ar}}\right), 131.3\left(\mathrm{~s}, C_{\mathrm{ar}}\right), 133.6\left(\mathrm{br}, C_{\mathrm{ar}}\right), 133.8\left(\mathrm{~d}, J_{\mathrm{CP}}=11.5 \mathrm{~Hz}, C_{\mathrm{ar}}\right)$, $134.0\left(\mathrm{~d}, J_{\mathrm{CP}}=13.7 \mathrm{~Hz}, C_{\mathrm{ar}}\right), 134.5\left(\mathrm{~d}, J_{\mathrm{CP}}=6.1 \mathrm{~Hz}, C_{\mathrm{ar}}\right), 135.1\left(\mathrm{br}, C_{\mathrm{ar}}\right), 135.6(\mathrm{br}$, $\left.C_{\mathrm{ar}}\right), 136.7\left(\mathrm{~s}, C_{\mathrm{ar}}\right), 141.8\left(\mathrm{~d}, J_{\mathrm{CP}}=6.0 \mathrm{~Hz}, C_{\mathrm{ar}}\right) ; \mathrm{EI}-\mathrm{MS} \mathrm{m} / \mathrm{z} 434\left[\mathrm{M}-\mathrm{CF}_{3} \mathrm{SO}_{3}\right]^{+}$.

Synthesis of phosphorus ylide 6: A 1:1 mixture of phosphonium and t-BuONa $(0.31$ mmol) was cooled to $-78^{\circ} \mathrm{C}$ and THF was added. The suspension was warmed to room temperature and stirred for $30 \mathrm{~min}$. All the volatiles were removed under vacuum and the red solid was extracted with 4x $20 \mathrm{~mL}$ of pentane. After evaporation of the solvent, the ylide $\mathbf{6}$ was obtained as a red powder. Recrystallisation in benzene at room temperature afforded the ylide as red crystals $(0.13 \mathrm{~g}, 95 \%): \mathrm{mp} 109-11{ }^{\circ} \mathrm{C}$ (dec); ${ }^{31} \mathrm{P}$ NMR $\left(\mathrm{THF}-d_{8}, 25^{\circ} \mathrm{C}\right) \delta-2.7 ;{ }^{1} \mathrm{H}$ NMR $\left(\mathrm{THF}-d_{8}, 25^{\circ} \mathrm{C}\right) \delta 1.10-1.55(\mathrm{~m}$, $\left.6 \mathrm{H}, \mathrm{CH}_{2}\right), 2.62\left(\mathrm{~m}, 2 \mathrm{H}, \mathrm{CH}_{2} \mathrm{~N}\right), 3.39\left(\mathrm{~m}, 2 \mathrm{H}, \mathrm{CH}_{2} \mathrm{~N}\right), 6.43\left(\mathrm{t}_{\text {like }}, 1 \mathrm{H}, J_{\mathrm{HH}}=7.2 \mathrm{~Hz}\right.$, $\left.\mathrm{CH}_{\mathrm{ar}}\right), 6.96\left(\mathrm{t}_{\text {like }}, 1 \mathrm{H}, J_{\mathrm{HH}}=7.2 \mathrm{~Hz}, \mathrm{CH}_{\mathrm{ar}}\right), 7.11\left(\mathrm{t}_{\text {like }}, 1 \mathrm{H}, J_{\mathrm{HH}}=7.2 \mathrm{~Hz}, \mathrm{CH}_{\mathrm{ar}}\right), 7.24(\mathrm{~d}$, $\left.1 \mathrm{H}, J_{\mathrm{HH}}=8.4 \mathrm{~Hz}, \mathrm{CH}_{\mathrm{ar}}\right), 7.45-7.56\left(\mathrm{~m}, 8 \mathrm{H}, \mathrm{CH}_{\mathrm{ar}}\right), 7.68\left(\mathrm{~m}, 4 \mathrm{H}, \mathrm{CH}_{\mathrm{ar}}\right), 8.02(\mathrm{~d}, 1 \mathrm{H}$, $\left.J_{\mathrm{HH}}=8.4 \mathrm{~Hz}, \mathrm{CH}_{\mathrm{ar}}\right), 8.35\left(\mathrm{dd}, 1 \mathrm{H}, J_{\mathrm{HH}}=8.4 \mathrm{~Hz}, J_{\mathrm{PH}}=5.2 \mathrm{~Hz}, \mathrm{CH}_{\mathrm{ar}}\right) ;{ }^{13} \mathrm{C}$ NMR (THF$\left.d_{8}, 25^{\circ} \mathrm{C}\right) \delta 24.3\left(\mathrm{~s}, \mathrm{CH}_{2}\right), 27.4\left(\mathrm{~s}, \mathrm{CH}_{2}\right), 53.3\left(\mathrm{~d}, J_{\mathrm{CP}}=2.5 \mathrm{~Hz}, \mathrm{CH}_{2} \mathrm{~N}\right), 69.1\left(\mathrm{~d}, J_{\mathrm{CP}}=\right.$ 
$\left.152.0 \mathrm{~Hz}, C_{\text {ylide }}\right), 112.5$ (s, $\left.C \mathrm{H}_{\mathrm{ar}}\right), 112.8$ (d, $\left.J_{\mathrm{CP}}=78.7 \mathrm{~Hz}, C_{\mathrm{ar}}\right), 116.4\left(\mathrm{~d}, J_{\mathrm{CP}}=4.2 \mathrm{~Hz}\right.$, $\left.C_{\mathrm{ar}}\right), 119.9\left(\mathrm{~d}, J_{\mathrm{CP}}=14.1 \mathrm{~Hz}, C_{\mathrm{ar}}\right), 123.5\left(\mathrm{~d}, J_{\mathrm{CP}}=9.0 \mathrm{~Hz}, C_{\mathrm{ar}}\right), 123.6\left(\mathrm{~d}, J_{\mathrm{CP}}=10.9\right.$ $\left.\mathrm{Hz}, C_{\mathrm{ar}}\right), 124.5\left(\mathrm{~s}, \mathrm{CH}_{\mathrm{ar}}\right), 127.3\left(\mathrm{~d}, J_{\mathrm{CP}}=2.7 \mathrm{~Hz}, C_{\mathrm{ar}}\right), 128.0\left(\mathrm{~d}, J_{\mathrm{CP}}=11.9 \mathrm{~Hz}\right.$, $\left.C \mathrm{H}_{\mathrm{ar}}\right), 129.4\left(\mathrm{~d}, J_{\mathrm{CP}}=1.9 \mathrm{~Hz}, C \mathrm{H}_{\mathrm{ar}}\right), 130.6\left(\mathrm{~d}, J_{\mathrm{CP}}=2.8 \mathrm{~Hz}, C \mathrm{H}_{\mathrm{ar}}\right), 131.4\left(\mathrm{~d}, J_{\mathrm{CP}}=88.5\right.$ $\left.\mathrm{Hz}, C_{\mathrm{ar}}\right), 132.2\left(\mathrm{~d}, J_{\mathrm{CP}}=6.0 \mathrm{~Hz}, C_{\mathrm{ar}}\right), 132.9\left(\mathrm{~d}, J_{\mathrm{CP}}=10.2 \mathrm{~Hz}, C_{\mathrm{ar}}\right), 139.5$ (d, $J_{\mathrm{CP}}=$ $\left.6.0 \mathrm{~Hz}, C_{\mathrm{ar}}\right), 145.5\left(\mathrm{~d}, J_{\mathrm{CP}}=35.2 \mathrm{~Hz}, C_{\mathrm{ar}}\right) ; \mathrm{MS}\left(\mathrm{DCI} / \mathrm{NH}_{3}\right) 434[\mathrm{M}+\mathrm{H}]^{+}$

Synthesis of Pd(II) complex 7. A 1:1 molar mixture of cyclic phosphonium triflate 5 and $t \mathrm{BuONa}(0.77 \mathrm{mmol})$ was cooled to $-78{ }^{\circ} \mathrm{C}$ and $20 \mathrm{~mL}$ of THF was added. The suspension was warmed to room temperature and stirred for $30 \mathrm{~min}$. All volatiles were removed under vacuum, then the red residue was dissolved in THF $(10 \mathrm{~mL})$, and added to a THF solution $(20 \mathrm{~mL})$ of $[\mathrm{Pd}(\mathrm{allyl}) \mathrm{Cl}]_{2}(0.14 \mathrm{~g}, 0.38 \mathrm{mmol})$ at $-78{ }^{\circ} \mathrm{C}$. The solution was allowed to warm to room temperature and stirred for $2 \mathrm{~h}$. After evaporation of the solvent, the solid was washed with $2 \times 20 \mathrm{~mL}$ of $\mathrm{Et}_{2} \mathrm{O}$ and extracted with $20 \mathrm{~mL}$ of $\mathrm{CH}_{2} \mathrm{Cl}_{2}$. After evaporation of the solvent, the yellow powder was recrystallized from THF at $-20{ }^{\circ} \mathrm{C}$, affording complex 7 (1/1.3 mixture of diastereomers) as pale yellow crystals (0.44g, 78\%): mp 190-192 ${ }^{\circ} \mathrm{C}$ (dec); ${ }^{31} \mathrm{P}$ NMR $\left(\mathrm{CDCl}_{3}, 25{ }^{\circ} \mathrm{C}\right) \delta+20.8,+19.8 ;{ }^{1} \mathrm{H}$ NMR $\left(\mathrm{CDCl}_{3}, 25{ }^{\circ} \mathrm{C}\right) \delta 0.91\left(\mathrm{~m}, 2 \mathrm{H}, \mathrm{CH}_{2}\right), 1.34$ (m, 1H, CH $\mathrm{CH}_{2}, 1.51$ (m, 5H, CH $), 1.68\left(\mathrm{~m}, 2 \mathrm{H}, \mathrm{CH}_{2}\right), 1.99\left(\mathrm{~m}, 2 \mathrm{H}, \mathrm{CH}_{2}\right), 2.56(\mathrm{~d}, 1 \mathrm{H}$, $\left.J_{\mathrm{HH}}=13.9 \mathrm{~Hz}, \mathrm{CH}_{\text {2allyl }}\right), 2.93$ (dd, $\left.1 \mathrm{H}, J_{\mathrm{HH}}=12.8 \mathrm{~Hz}, J_{\mathrm{PH}}=9.7 \mathrm{~Hz}, \mathrm{CH}_{2 \text { allyl }}\right), 3.11$ (d, $\left.1 \mathrm{H}, J_{\mathrm{HH}}=13.3 \mathrm{~Hz}, \mathrm{CH}_{2 \mathrm{allyl}}\right), 3.36\left(\mathrm{~m}, 2 \mathrm{H}, \mathrm{CH}_{2}\right), 3.41\left(\mathrm{dd}, 1 \mathrm{H}, J_{\mathrm{HH}}=13.3 \mathrm{~Hz}, J_{\mathrm{PH}}=9.6\right.$ $\left.\mathrm{Hz}, \mathrm{CH}_{\text {2allyl }}\right), 3.50\left(\mathrm{~m}, 2 \mathrm{H}, \mathrm{CH}_{2}\right), 3.62\left(\mathrm{dd}, 1 \mathrm{H}, J_{\mathrm{HH}}=2.5 \mathrm{~Hz}, J_{\mathrm{HH}}=8.5 \mathrm{~Hz}, \mathrm{CH}_{\text {2allyl }}\right)$, $4.05\left(\mathrm{~d}, 1 \mathrm{H}, J_{\mathrm{HH}}=8.5 \mathrm{~Hz}, \mathrm{CH}_{\text {2allyl }}\right), 4.10\left(\mathrm{~m}, 2 \mathrm{H}, \mathrm{CH}_{2}\right), 4.26\left(\mathrm{~m}, 2 \mathrm{H}, \mathrm{CH}_{2}\right), 4.46$ (dd, $\left.1 \mathrm{H}, J_{\mathrm{HH}}=5.9 \mathrm{~Hz}, J_{\mathrm{PH}}=6.0 \mathrm{~Hz}, \mathrm{CH}_{\text {2allyl }}\right), 4.56\left(\mathrm{dd}, 1 \mathrm{H}, J_{\mathrm{HH}}=5.9 \mathrm{~Hz}, J_{\mathrm{PH}}=6.0 \mathrm{~Hz}\right.$, $\left.\mathrm{CH}_{\text {2allyl }}\right), 5.44\left(\mathrm{~m}, 1 \mathrm{H}, \mathrm{CH}_{\text {allyl }}\right), 5.69\left(\mathrm{~m}, 1 \mathrm{H}, \mathrm{CH}_{\text {allyl }}\right), 6.56\left(\mathrm{~d}, 1 \mathrm{H}, J_{\mathrm{HH}}=7.7 \mathrm{~Hz}, \mathrm{CH}_{\text {ar }}\right)$, $6.67\left(\mathrm{~d}, 2 \mathrm{H}, J_{\mathrm{HH}}=7.6 \mathrm{~Hz}, \mathrm{CH}_{\mathrm{ar}}\right), 6.74\left(\mathrm{dd}, 1 \mathrm{H}, J_{\mathrm{HH}}=7.7 \mathrm{~Hz}, J_{\mathrm{PH}}=10.4 \mathrm{~Hz}, \mathrm{CH}_{\mathrm{ar}}\right)$, $6.78\left(\mathrm{~d}, 1 \mathrm{H}, J_{\mathrm{HH}}=8.4 \mathrm{~Hz}, \mathrm{CH} H_{\mathrm{ar}}\right), 6.84\left(\mathrm{dd}, 1 \mathrm{H}, J_{\mathrm{HH}}=8.3 \mathrm{~Hz}, J_{\mathrm{PH}}=10.5 \mathrm{~Hz}, \mathrm{CH}_{\mathrm{ar}}\right)$, $6.92\left(\mathrm{t}_{\text {like, }}, 1 \mathrm{H}, J_{\mathrm{HH}}=7.6 \mathrm{~Hz}, \mathrm{CH}_{\mathrm{ar}}\right), 7.00\left(\mathrm{t}_{\text {like }}, 1 \mathrm{H}, J_{\mathrm{HH}}=7.1 \mathrm{~Hz}, \mathrm{CH}_{\mathrm{ar}}\right), 7.03-7.55(\mathrm{~m}$, $\left.26 \mathrm{H}, \mathrm{CH}_{\mathrm{ar}}\right), 7.57\left(\mathrm{t}_{\text {like }}, 2 \mathrm{H}, J_{\mathrm{HH}}=7.5 \mathrm{~Hz}, \mathrm{CH}_{\mathrm{ar}}\right) ;{ }^{13} \mathrm{C} \mathrm{NMR}\left(\mathrm{CDCl}_{3}, 25{ }^{\circ} \mathrm{C}\right) \delta 23.1(\mathrm{~s}$, $\mathrm{CH}_{2}$ ), 23.2 (s, $\mathrm{CH}_{2}$ ), 26.7 (s, $\mathrm{CH}_{2}$ ), 26.8 (s, $\mathrm{CH}_{2}$ ), 26.9 (s, $\mathrm{CH}_{2}$ ), 27.0 (s, $\mathrm{CH}_{2}$ ), 54.7 (s, $\left.\mathrm{CH}_{2} \mathrm{~N}\right), 54.8\left(\mathrm{~s}, \mathrm{CH}_{2} \mathrm{~N}\right), 61.9\left(\mathrm{~d}, J_{\mathrm{CP}}=28.2 \mathrm{~Hz}, \mathrm{CH}_{2 \text { allyl }}\right), 63.3\left(\mathrm{~d}, J_{\mathrm{CP}}=28.9 \mathrm{~Hz}\right.$, $\mathrm{CH}_{\text {2allyl }}$ ), 64.3 (s, $\left.\mathrm{CH}_{2} \mathrm{~N}\right), 64.5\left(\mathrm{~s}, \mathrm{CH}_{2} \mathrm{~N}\right), 66.5$ (s, $\mathrm{CH}_{\text {2allyl }}$ ), 67.2 (s, $\left.\mathrm{CH}_{\text {2allyl }}\right), 120.5$ (s, $\left.C_{\mathrm{ar}}\right), 120.8\left(\mathrm{~d}, J_{\mathrm{CP}}=4.8 \mathrm{~Hz}, C_{\mathrm{allyl}}\right), 120.9\left(\mathrm{q}, J_{\mathrm{CF}}=320.9 \mathrm{~Hz}, C_{3} \mathrm{SO}_{3}\right), 121.0\left(\mathrm{~s}, C_{\mathrm{ar}}\right)$, $121.7\left(\mathrm{~d}, J_{\mathrm{CP}}=5.1 \mathrm{~Hz}, C \mathrm{H}_{\mathrm{allyl}}\right), 127.8\left(\mathrm{~d}, J_{\mathrm{CP}}=7.4 \mathrm{~Hz}, C_{\mathrm{ar}}\right), 128.0\left(\mathrm{~d}, J_{\mathrm{CP}}=7.5 \mathrm{~Hz}\right.$, $\left.C_{\mathrm{ar}}\right), 128.4\left(\mathrm{~s}, C_{\mathrm{ar}}\right), 128.5\left(\mathrm{~s}, C_{\mathrm{ar}}\right), 128.6\left(\mathrm{~s}, C_{\mathrm{ar}}\right), 128.8\left(\mathrm{~d}, J_{\mathrm{CP}}=11.5 \mathrm{~Hz}, C_{\mathrm{ar}}\right), 128.9$ (s, $\left.C_{\mathrm{ar}}\right), 129.0\left(\mathrm{~d}, J_{\mathrm{CP}}=13.0 \mathrm{~Hz}, C_{\mathrm{ar}}\right), 129.4\left(\mathrm{~d}, J_{\mathrm{CP}}=10.8 \mathrm{~Hz}, C_{\mathrm{ar}}\right), 129.5\left(\mathrm{~d}, J_{\mathrm{CP}}=10.8\right.$ $\left.\mathrm{Hz}, C_{\mathrm{ar}}\right), 129.9\left(\mathrm{~d}, J_{\mathrm{CP}}=44.7 \mathrm{~Hz}, C_{\mathrm{ar}}\right), 130.6\left(\mathrm{~d}, J_{\mathrm{CP}}=19.8 \mathrm{~Hz}, C_{\mathrm{ar}}\right), 130.7\left(\mathrm{~s}, C_{\mathrm{ar}}\right)$, $130.9\left(\mathrm{~d}, J_{\mathrm{CP}}=34.5 \mathrm{~Hz}, C_{\mathrm{ar}}\right), 131.0\left(\mathrm{~s}, C_{\mathrm{ar}}\right), 131.4\left(\mathrm{~s}, C_{\mathrm{ar}}\right), 131.6\left(\mathrm{~d}, J_{\mathrm{CP}}=1.5 \mathrm{~Hz}, C_{\mathrm{ar}}\right)$, $131.7\left(\mathrm{~d}, J_{\mathrm{CP}}=2.0 \mathrm{~Hz}, C_{\mathrm{ar}}\right), 131.8\left(\mathrm{~s}, C_{\mathrm{ar}}\right), 131.9\left(\mathrm{~s}, C_{\mathrm{ar}}\right), 132.6\left(\mathrm{~d}, J_{\mathrm{CP}}=7.4 \mathrm{~Hz}, C_{\mathrm{ar}}\right)$, $132.7\left(\mathrm{~d}, J_{\mathrm{CP}}=7.6 \mathrm{~Hz}, C_{\mathrm{ar}}\right), 133.3\left(\mathrm{~d}, J_{\mathrm{CP}}=14.3 \mathrm{~Hz}, C_{\mathrm{ar}}\right), 133.7\left(\mathrm{~d}, J_{\mathrm{CP}}=14.9 \mathrm{~Hz}, C_{\mathrm{ar}}\right)$, $134.7\left(\mathrm{~d}, J_{\mathrm{CP}}=14.4 \mathrm{~Hz}, C_{\mathrm{ar}}\right), 134.9\left(\mathrm{~d}, J_{\mathrm{CP}}=14.7 \mathrm{~Hz}, C_{\mathrm{ar}}\right), 140.0\left(\mathrm{~s}, C_{\mathrm{ar}}\right), 140.5(\mathrm{~s}$, $\left.C_{\mathrm{ar}}\right), 144.0\left(\mathrm{~d}, J_{\mathrm{CP}}=12.9 \mathrm{~Hz}, C_{\mathrm{ar}}\right), 241.5\left(\mathrm{~d}, J_{\mathrm{CP}}=19.4 \mathrm{~Hz}, C_{\text {carbene }}\right), 242.7\left(\mathrm{~d}, J_{\mathrm{CP}}=\right.$ $\left.19.1 \mathrm{~Hz}, C_{\text {carbene }}\right)$; $\mathrm{MS}(\mathrm{FAB}+) 580[\mathrm{M}]^{+}$.

Synthesis of $\mathbf{R h}(\mathbf{I})$ complex 8. Using the same experimental procedure as above, but with $[\mathrm{RhCl}(\mathrm{cod})]_{2}(0.84 \mathrm{~g}, 0.17 \mathrm{mmol})$, and stirring for 3 days, Rh(I) complex 8 was obtained by slow crystallization from a THF/hexane solution as orange crystals $(0.23$ g, 86\%): m.p. $215-217{ }^{\circ} \mathrm{C}$ (dec); ${ }^{31} \mathrm{P}$ NMR $\left(\mathrm{CDCl}_{3}, 25{ }^{\circ} \mathrm{C}\right) \delta+20.3\left(\mathrm{~d}, J_{\mathrm{PRh}}=173.1\right.$ 
$\mathrm{Hz}) ;{ }^{1} \mathrm{H}$ NMR $\left(\mathrm{CDCl}_{3}, 25{ }^{\circ} \mathrm{C}\right) \delta 0.84\left(\mathrm{~m}, 1 \mathrm{H}, \mathrm{CH}_{2}\right), 1.36\left(\mathrm{~m}, 1 \mathrm{H}, \mathrm{CH}_{2}\right), 1.51(\mathrm{~m}, 2 \mathrm{H}$, $\left.\mathrm{CH}_{2}\right), 1.67\left(\mathrm{~m}, 1 \mathrm{H}, \mathrm{CH}_{2}\right), 1.94\left(\mathrm{~m}, 1 \mathrm{H}, \mathrm{CH}_{2}\right), 2.07$ (m, 2H, $\left.\mathrm{CH}_{2}\right), 2.20\left(\mathrm{~m}, 1 \mathrm{H}, \mathrm{CH}_{2}\right)$, $2.38\left(\mathrm{~m}, 2 \mathrm{H}, \mathrm{CH}_{2}\right), 2.46\left(\mathrm{~m}, 1 \mathrm{H}, \mathrm{CH}_{2}\right), 2.56\left(\mathrm{~m}, 1 \mathrm{H}, \mathrm{CH}_{2}\right), 2.64\left(\mathrm{~m}, 1 \mathrm{H}, \mathrm{CH}_{2}\right), 3.14$ $\left(\mathrm{m}, 1 \mathrm{H}, \mathrm{CH}_{2} \mathrm{~N}\right), 3.42\left(\mathrm{~m}, 1 \mathrm{H}, \mathrm{CH}_{2} \mathrm{~N}\right), 3.65\left(\mathrm{~m}, 1 \mathrm{H}, \mathrm{CH}_{\text {cod }}\right), 4.09\left(\mathrm{~m}, 1 \mathrm{H}, \mathrm{CH}_{\text {cod }}\right), 4.72$ $\left(\mathrm{m}, 2 \mathrm{H}, \mathrm{CH}_{2} \mathrm{~N}\right), 5.10\left(\mathrm{~m}, 1 \mathrm{H}, \mathrm{CH}_{\mathrm{cod}}\right), 5.45\left(\mathrm{~m}, 1 \mathrm{H}, \mathrm{CH}_{\mathrm{cod}}\right), 6.34\left(\mathrm{~d}, 1 \mathrm{H}, J_{\mathrm{HH}}=7.6 \mathrm{~Hz}\right.$, $\left.\mathrm{CH}_{\mathrm{ar}}\right), 6.89\left(\mathrm{~d}, 1 \mathrm{H}, J_{\mathrm{HH}}=7.6 \mathrm{~Hz}, \mathrm{CH}_{\mathrm{ar}}\right), 7.02\left(\mathrm{~m}, 2 \mathrm{H}, \mathrm{CH}_{\mathrm{ar}}\right), 7.41\left(\mathrm{~m}, 14 \mathrm{H}, \mathrm{CH}_{\mathrm{ar}}\right) ;{ }^{13} \mathrm{C}$ NMR $\left(\mathrm{CDCl}_{3}, 25{ }^{\circ} \mathrm{C}\right) \delta 23.1$ (s, $\left.\mathrm{CH}_{2}\right), 26.0\left(\mathrm{~s}, \mathrm{CH}_{2}\right), 27.0\left(\mathrm{~s}, \mathrm{CH}_{2}\right), 28.6\left(\mathrm{~s}, \mathrm{CH}_{2 \mathrm{cod}}\right)$, 31.4 (s, $\mathrm{CH}_{2 \mathrm{cod}}$ ), $54.8\left(\mathrm{~s}, \mathrm{CH}_{2} \mathrm{~N}\right), 62.5\left(\mathrm{~s}, \mathrm{CH}_{2} \mathrm{~N}\right), 92.7$ (d, J $\left.\mathrm{JRh}=5.5 \mathrm{~Hz}, \mathrm{CH}_{\mathrm{cod}}\right), 97.2$ (dd, $\left.J_{\mathrm{CRh}}=8.4 \mathrm{~Hz}, J_{\mathrm{CP}}=8.4 \mathrm{~Hz}, C_{\mathrm{cod}}\right), 98.3\left(\mathrm{dd}, J_{\mathrm{CRh}}=8.4 \mathrm{~Hz}, J_{\mathrm{CP}}=8.4 \mathrm{~Hz}, C_{\mathrm{cod}}\right.$ ), 99.0 (d, $\left.J_{\mathrm{CRh}}=6.3 \mathrm{~Hz}, C_{\mathrm{cod}}\right), 120.8$ (s, $\left.C_{\mathrm{ar}}\right), 121.0$ (q, $\left.J_{\mathrm{CF}}=320.9 \mathrm{~Hz}, C_{3} \mathrm{SO}_{3}\right), 127.4$ $\left(\mathrm{d}, J_{\mathrm{CP}}=41.3 \mathrm{~Hz}, C_{\mathrm{ar}}\right), 127.7\left(\mathrm{~d}, J_{\mathrm{CP}}=6.8 \mathrm{~Hz}, C_{\mathrm{ar}}\right), 128.5\left(\mathrm{~s}, C_{\mathrm{ar}}\right), 128.6\left(\mathrm{~d}, J_{\mathrm{CP}}=9.8\right.$ $\left.\mathrm{Hz}, C_{\mathrm{ar}}\right), 129.1$ (d, $\left.J_{\mathrm{CP}}=4.1 \mathrm{~Hz}, C_{\mathrm{ar}}\right), 129.2\left(\mathrm{~s}, C_{\mathrm{ar}}\right), 129.3$ (d, $\left.J_{\mathrm{CP}}=9.9 \mathrm{~Hz}, C_{\mathrm{ar}}\right), 130.4$ (s, $C_{\mathrm{ar}}$ ), 131.1 (d, $\left.J_{\mathrm{CP}}=1.8 \mathrm{~Hz}, C_{\mathrm{ar}}\right), 131.3\left(\mathrm{~s}, C_{\mathrm{ar}}\right), 132.1$ (d, $\left.J_{\mathrm{CP}}=43.2 \mathrm{~Hz}, C_{\mathrm{ar}}\right), 133.0$ $\left(\mathrm{d}, J_{\mathrm{CP}}=6.9 \mathrm{~Hz}, C_{\mathrm{ar}}\right), 133.2\left(\mathrm{~d}, J_{\mathrm{CP}}=11.9 \mathrm{~Hz}, C_{\mathrm{ar}}\right), 135.9$ (d, $\left.J_{\mathrm{CP}}=12.9 \mathrm{~Hz}, C_{\mathrm{ar}}\right), 139.8$ (d, $\left.J_{\mathrm{CP}}=1.6 \mathrm{~Hz}, C_{\mathrm{ar}}\right), 144.3\left(\mathrm{~d}, J_{\mathrm{CP}}=12.6 \mathrm{~Hz}, C_{\mathrm{ar}}\right), 249.3\left(\mathrm{dd}, J_{\mathrm{CRh}}=37.5 \mathrm{~Hz}, J_{\mathrm{CP}}=\right.$ $\left.12.0 \mathrm{~Hz}, C_{\text {carbene }}\right)$; MS (FAB+) $644[\mathrm{M}]^{+}$.

References:

1 (a) Canac, Y.; Conejero, S.; Soleilhavoup, M.; Donnadieu, B.; Bertrand, G. J. Am. Chem. Soc. 2006, 128, 459. (b) Conejero, S.; Song, M.; Martin, D.; Canac, Y.; Soleilhavoup, M.; Bertrand, G. Chem. Asian J. 2006, 1, 155.

2 Oba, M.; Iida, M.; Nishiyama, K. Organometallics 2001, 20, 4287. 